\title{
FIRST ORDER DEFORMATIONS OF PAIRS AND NON-EXISTENCE OF RATIONAL CURVES
}

\author{
BIN WANG
}

\begin{abstract}
Let $X_{0}$ be a smooth hypersurface (assumed not to be generic) in projective space $\mathbf{P}^{n}, n \geq 4$, over complex numbers, and $C_{0}$ a smooth rational curve on $X_{0}$. We are interested in deformations of the pair $C_{0}$ and $X_{0}$. In this paper, we prove that, if the first order deformations of the pair exist along each deformation of the hypersurface $X_{0}$,
\end{abstract} then $\operatorname{deg}\left(C_{0}\right)$ cannot be in the range

$$
\left(m \frac{2 \operatorname{deg}\left(X_{0}\right)+1}{\operatorname{deg}\left(X_{0}\right)+1}, \frac{2+m(n-2)}{2 n-\operatorname{deg}\left(X_{0}\right)-1}\right),
$$

where $m$ is any non negative integer less than

$$
\operatorname{dim}\left(\left.H^{0}\left(\mathcal{O}_{\mathbf{P}^{n}}(1)\right)\right|_{C_{0}}\right)-1 .
$$

1. Introduction. Throughout the paper, varieties are over the complex numbers. We are interested in conditions on the first order deformations of a pair of a rational curve and a hypersurface. So let us introduce the first order condition.

Let $H^{0}\left(\mathcal{O}_{\mathbf{P}^{n}}(h)\right)$ denote the vector space of homogeneous polynomials of degree $h$ in $n+1$ variables with $n \geq 4$. Let $f_{0} \in H^{0}\left(\mathcal{O}_{\mathbf{P}^{n}}(h)\right)$ be such that

$$
X_{0}=\operatorname{div}\left(f_{0}\right)
$$

is a smooth hypersurface. Let

$$
\left[f_{0}\right] \in \mathbf{P}\left(H^{0}\left(\mathcal{O}_{\mathbf{P}^{n}}(h)\right)\right)
$$

denote the corresponding point of $f_{0}$ in the projectivization. Let

$$
c_{0}: \mathbf{P}^{1} \longrightarrow X_{0} \subset \mathbf{P}^{n}
$$

2010 AMS Mathematics subject classification. Primary 14J70, 14N10, 14 N25.

Keywords and phrases. Hypersurface, rational curve, normal bundle.

Received by the editors on January 27, 2012, and in revised form on June 15, 2014. 
be a smooth embedding of $\mathbf{P}^{1}$, whose image is $C_{0}$. Let

$$
\mathbb{H}^{1}\left(T_{X_{0}} \longrightarrow N_{C_{0} / X_{0}}\right)
$$

be the hypercohomology of the complex that is isomorphic to the tangent space of the deformations of the pair $C_{0} \subset X_{0}$ and $H^{1}\left(T_{X_{0}}\right)$, the space that is isomorphic to the tangent space of the moduli space of hypersurfaces at the point $X_{0}$. There is a known exact sequence:

$$
\mathbb{H}^{1}\left(T_{X_{0}} \longrightarrow N_{C_{0} / X_{0}}\right) \stackrel{\phi}{\longrightarrow} H^{1}\left(T_{X_{0}}\right) \quad \longrightarrow H^{1}\left(N_{C_{0} / X_{0}}\right) .
$$

Theorem 1.1. Let $\mu\left(C_{0}\right)=\operatorname{dim}\left(c_{0}^{*}\left(H^{0}\left(\mathcal{O}_{\mathbf{P}^{n}}(1)\right)\right)\right)$.

(i) If

$$
\phi \text { is surjective, }
$$

then $\operatorname{deg}\left(C_{0}\right)$ cannot be in the range

$$
\left(m \frac{2 h+1}{h+1}, \frac{2+m(n-2)}{2 n-h-1}\right)
$$

in the case

$$
m \frac{2 h+1}{h+1}<\frac{2+m(n-2)}{2 n-h-1}
$$

where $m$ is any non negative integer less than $\mu\left(C_{0}\right)-1$. It follows that, if assumption (1.3) holds, $\operatorname{deg}\left(C_{0}\right)$ cannot be in the range

$$
\left(1, \frac{n}{2 n-h-1}\right), \quad \text { for } m=1
$$

in the case $n-h-1<0$.

In particular,

(ii) if

$$
H^{1}\left(N_{C_{0} / X_{0}}\right)=0,
$$

all results in part (i) hold;

(iii) if $X_{0}$ is a generic hypersurface and contains a rational curve $C_{0}$, all results in part (i) hold.

1.1. Remark. The result in this theorem is new but is related to many previously known results by Clemens $[\mathbf{1}, \mathbf{2}, \mathbf{3}]$, Chiantini, Lopez and Ran [4], Ein [5], Katz [6], Pacienza [8], Voisin [9, 10] and Xu [12], 
etc. ${ }^{1}$ They all studied pairs of rational curves (or higher dimensional subvarieties) and generic hypersurfaces of a certain smooth projective variety. The study of the exact sequence (1.2) appeared in many previous papers above. But here we use it as the only assumption for our result.

To explain in detail how we utilize assumption (1.3) at a nongeneric hypersurface $f_{0}$, we let $M_{d}$ be the parameter space of smooth embeddings $\mathbf{P}^{1} \rightarrow \mathbf{P}^{n}$, whose image has degree $d$. So $M_{d}$ is an open set of

$$
\mathbf{P}\left(\oplus_{n+1} \mathcal{O}_{\mathbf{P}^{n}}(d)\right) .
$$

The smooth embedding map $c_{0}$ represents a point in $M_{d}$ which is still denoted by $c_{0}$. Let

$$
\begin{gathered}
\Gamma \subset M_{d} \times \mathbf{P}\left(H^{0}\left(\mathcal{O}_{\mathbf{P}^{n}}(h)\right)\right) \\
\Gamma=\left\{(c,[f]): c^{*}(f)=0\right\} .
\end{gathered}
$$

Then the assumption in part (iii) that $X_{0}$ is generic is equivalent to the assumption that there is an irreducible component $\Gamma_{0}$ of $\Gamma$ dominating

$$
\mathbf{P}\left(H^{0}\left(\mathcal{O}_{\mathbf{P}^{n}}(h)\right)\right),
$$

and $\left(c_{0},\left[f_{0}\right]\right)$ is generic in $\Gamma_{0}$. Then it is well known that this implies the surjectivity of $\phi$ at $\left(c_{0},\left[f_{0}\right]\right)$, which is our assumption (1.3) at this point (see Lemma 2.2 below). The converse may not be true, i.e., the surjectivity of $\phi\left(\right.$ at $\left.\left(c_{0},\left[f_{0}\right]\right)\right)$ may not imply the existence of $\Gamma_{0}$ containing the fixed point $\left(c_{0},\left[f_{0}\right]\right)$.

1.2. Idea of the proof. The main idea of the proof is to use special pencils of hypersurfaces constructed from general plane sections $L_{i}$ (the pencil of $L_{1} \cdots L_{h}$ and $f_{0}$ ). If the rational curve $C_{0}$ can deform to all hypersurfaces in first order, i.e., assumption (1.3) holds, then the collection $V$ of the first order deformations of the rational curve along the directions of all such pencils generates some rank-2 quotient bundle $c_{0}^{*}\left(T_{\mathbf{P}^{n}}\right) / E^{\prime}$ (see subsection 3.2). But, if the numerical condition on $C_{0}$,

$$
m\left(\frac{2 h+1}{h+1}\right) \leq \operatorname{deg}\left(C_{0}\right) \leq \frac{2+m(n-2)}{2 n-h-1},
$$

is satisfied, $V$ fails to generate $c_{0}^{*}\left(T_{\mathbf{P}^{n}}\right) / E^{\prime}$ because all the first order deformations in $V$ lie in a fixed, proper sub-bundle of $c_{0}^{*}\left(T_{\mathbf{P}^{n}}\right) / E^{\prime}$. The failure is forced by the numerical bounds (1.5) (through carefully 
designed first order deformations of the hypersurface constructed from the pencils above). Thus, the numerical bounds (1.5) contradict the first order deformation assumption (1.3).

In Section 2, we give another description of assumption (1.3) on first order deformations of the pair. In Section 3, we give the property on the global generation of the bundle $N_{C_{0} / \mathbf{P}^{n}}$. This property, Proposition 3.3, requires a long set-up. In Section 4, we show that the numerical condition (1.5) forces the property on the global generation, Proposition 3.3, to fail. This proves Theorem 1.1. In Section 5, we give three examples for Theorem 1.1.

2. First order deformations of the pair. In this section, we give another description of assumption (1.3). Let

$$
S \subset \mathbf{P}\left(H^{0}\left(\mathcal{O}_{\mathbf{P}^{n}}(h)\right)\right)
$$

be an irreducible subvariety (quasi or projective) that contains $\left[f_{0}\right]$ and is smooth at $\left[f_{0}\right]$ (so $S$ could be a Zariski open set). Let

$$
\mathcal{X}_{S} \subset \mathbf{P}^{n} \times S,
$$

and

$$
\mathcal{X}_{S}=\{(x,[f]):[f] \in S, f(x)=0\},
$$

be the universal hypersurface for $S \subset \mathbf{P}\left(H^{0}\left(\mathcal{O}_{\mathbf{P}^{n}}(h)\right)\right)$.

Let

$$
\begin{aligned}
\bar{c}_{0}: \mathbf{P}^{1} & \longrightarrow C_{0} \times\left\{\left[f_{0}\right]\right\} \subset \mathcal{X}_{S} \\
t & \longrightarrow\left(c_{0}(t),\left[f_{0}\right]\right)
\end{aligned}
$$

be the smooth embedding determined by the above embedding $c_{0}$. The projection

$$
P_{S}: \mathcal{X}_{S} \longrightarrow S
$$

has a differential map

$$
T_{\left(q,\left[f_{0}\right]\right)} \mathcal{X}_{S} \longrightarrow T_{\left[f_{0}\right]} S, \quad q \in C_{0},
$$

which can be extended to a bundle map

$$
\left(P_{S}\right)_{*}: \bar{c}_{0}^{*}\left(T_{\mathcal{X}_{S}}\right) \longrightarrow T_{\left[f_{0}\right]} S \otimes \mathcal{O}_{\mathbf{P}^{1}} .
$$


At last, we obtain a homomorphism on the vector spaces

$$
P_{S}^{s}: H^{0}\left(\bar{c}_{0}^{*}\left(T_{\mathcal{X}_{S}}\right)\right) \longrightarrow T_{\left[f_{0}\right]} S,
$$

where $T_{\left[f_{0}\right]} S \simeq H^{0}\left(T_{\left[f_{0}\right]} S \otimes \mathcal{O}_{\mathbf{P}^{1}}\right)$ is the space of global sections of the trivial bundle, each of whose fibre is $T_{\left[f_{0}\right]} S$.

Now consider the diagram

$$
\begin{aligned}
& \mathbb{H}^{1}\left(T_{X_{0}} \rightarrow N_{C_{0} / X_{0}}\right) \\
& \downarrow \phi \\
& T_{\left[f_{0}\right]} \mathbf{P}\left(H^{0}\left(\mathcal{O}_{\mathbf{P}^{n}}(h)\right)\right) \stackrel{\psi}{\rightarrow} \quad H^{1}\left(T_{X_{0}}\right),
\end{aligned}
$$

where the map $\psi$ is the differential (surjective for $n \geq 4$ ) at $\left[f_{0}\right]$ from $\mathbf{P}\left(H^{0}\left(\mathcal{O}_{\mathbf{P}^{n}}(h)\right)\right.$ to the deformation space of complex structures of the differential manifold $X_{0}$.

\section{Lemma 2.1.}

$$
\psi\left(T_{\left[f_{0}\right]} S\right) \subset \text { image }(\phi)
$$

if and only if $P_{S}^{s}$ is surjective.

Proof. Let $M_{d}$ be the parameter space of smooth embedding $\mathbf{P}^{1} \rightarrow$ $\mathbf{P}^{n}$, whose image has degree $d$. So $M_{d}$ is an open set of

$$
\mathbf{P}\left(\oplus_{n+1} \mathcal{O}_{\mathbf{P}^{n}}(d)\right) .
$$

The map $c_{0}$ represents a point in $M_{d}$ which is still denoted by $c_{0}$. Let $\mathcal{X}_{n}$ be the universal hypersurface for $S=\mathbf{P}\left(H^{0}\left(\mathcal{O}_{\mathbf{P}^{n}}(h)\right)\right)$ (defined in formula (2.2)). Let

$$
\begin{aligned}
& \Gamma \subset M_{d} \times \mathbf{P}\left(H^{0}\left(\mathcal{O}_{\mathbf{P}^{n}}(h)\right)\right) \\
& \Gamma=\left\{(c,[f]): c^{*}(f)=0\right\}
\end{aligned}
$$

be the incidence scheme containing the point $\left(c_{0},\left[f_{0}\right]\right)$. Let $T_{\left(c_{0},\left[f_{0}\right]\right)} \Gamma$ be the Zariski tangent space of $\Gamma$. Let $e$ be the evaluation map

$$
\begin{aligned}
e: \Gamma \times \mathbf{P}^{1} & \longrightarrow \mathcal{X}_{n} \\
\quad(c,[f], t) & \longrightarrow(c(t),[f]) .
\end{aligned}
$$

Its differential map induces a bundle map

$$
e_{*}: T_{\left(c_{0},\left[f_{0}\right]\right)} \Gamma \otimes \mathcal{O}_{\mathbf{P}^{1}} \rightarrow c_{0}^{*}\left(T_{\mathcal{X}_{n}}\right) .
$$


It further induces a homomorphism on the cohomologies:

$$
e^{s}: T_{\left(c_{0},\left[f_{0}\right]\right)} \Gamma \longrightarrow H^{0}\left(c_{0}^{*}\left(T_{\mathcal{X}_{n}}\right)\right),
$$

where $T_{\left(c_{0},\left[f_{0}\right]\right)} \Gamma=H^{0}\left(T_{\left(c_{0},\left[f_{0}\right]\right)} \Gamma \otimes \mathcal{O}_{\mathbf{P}^{1}}\right)$. Also, there is a surjective map $\eta$ :

$$
T_{\left(C_{0},\left[f_{0}\right]\right)} \Gamma \quad \rightarrow \quad \mathbb{H}^{1}\left(T_{X_{0}} \rightarrow N_{C_{0} / X_{0}}\right),
$$

such that the following diagram commutes

$$
\begin{array}{ccccc}
T_{\left(C_{0},\left[f_{0}\right]\right)} \Gamma & = & T_{\left(C_{0},\left[f_{0}\right]\right)} \Gamma & \stackrel{\eta}{\longrightarrow} & \mathbb{H}^{1}\left(T_{X_{0}} \rightarrow N_{C_{0} / X_{0}}\right) \\
H^{0}\left(\bar{c}_{0}^{*}\left(T_{\mathcal{X}_{n}}\right)\right) & \stackrel{P_{n}^{s}}{\rightarrow} & T_{\left[f_{0}\right]} \mathbf{P}\left(H^{0}\left(\mathcal{O}_{\mathbf{P}^{n}}(h)\right)\right) & \stackrel{\psi}{\rightarrow} & H^{1}\left(T_{X_{0}}\right),
\end{array}
$$

where $P_{n}^{s}$ is the corresponding map in formula (2.3) for

$$
S=\mathbf{P}\left(H^{0}\left(\mathcal{O}_{\mathbf{P}^{n}}(h)\right)\right) .
$$

Because

$$
T_{c_{0}} M_{d} \longrightarrow H^{0}\left(c_{0}^{*}\left(T_{\mathbf{P}^{n}}\right)\right)
$$

is surjective (it is an isomorphism), $e^{s}$ has to be surjective. Then, the lemma is true for $S=\mathbf{P}\left(H^{0}\left(\mathcal{O}_{\mathbf{P}^{n}}(h)\right)\right)$.

Now we consider the subvariety $S \subset \mathbf{P}\left(H^{0}\left(\mathcal{O}_{\mathbf{P}^{n}}(h)\right)\right)$ in the lemma. If $\psi\left(T_{\left[f_{0}\right]} S\right) \subset$ image $(\phi)$, for any $\alpha \in T_{\left[f_{0}\right]} S$, we apply the diagram to find a section $\sigma \in H^{0}\left(\bar{c}_{0}^{*}\left(T_{\mathcal{X}_{n}}\right)\right)$ such that $P_{n}^{s}(\sigma)=\alpha$. Because $P_{n}^{s}(\sigma)=\alpha \in T_{\left[f_{0}\right]} S, \sigma$ must be in the subspace $H^{0}\left(\bar{c}_{0}^{*}\left(T_{\mathcal{X}_{S}}\right)\right)$ of $H^{0}\left(\bar{c}_{0}^{*}\left(T_{\mathcal{X}_{n}}\right)\right)$. Thus, $P_{S}^{s}$ is surjective. Conversely, we suppose $P_{S}^{s}$ is surjective. For any $\alpha \in T_{\left[f_{0}\right]} S$, using the commutative diagram, we obtain

$$
\psi(\alpha) \in \phi \circ \eta \circ\left(e^{s}\right)^{-1} \circ\left(P_{S}^{s}\right)^{-1}(\alpha)
$$

This completes the proof.

Lemma 2.2. If there is an irreducible component $\Gamma_{0}$ of the incidence scheme

$$
\left\{(c,[f]) \in M_{d} \times \mathbf{P}\left(H^{0}\left(\mathcal{O}_{\mathbf{P}^{n}}(h)\right)\right): c^{*}(f)=0\right\},
$$

such that $\Gamma_{0}$ dominates $\mathbf{P}\left(H^{0}\left(\mathcal{O}_{\mathbf{P}^{n}}(h)\right)\right)$ and $\left(c_{0},\left[f_{0}\right]\right) \in \Gamma_{0}$ is generic, then $\phi$ is surjective. 
Proof. In this proof, we consider the entire space of hypersurfaces, i.e.,

$$
S=\mathbf{P}\left(H^{0}\left(\mathcal{O}_{\mathbf{P}^{n}}(h)\right)\right)
$$

As before, $\mathcal{X}_{n}$ denotes the universal hypersurface corresponding to $\mathbf{P}\left(H^{0}\left(\mathcal{O}_{\mathbf{P}^{n}}(h)\right)\right)$.

Let $c_{0}$ be as above, and let

$$
\bar{c}_{0}: \mathbf{P}^{1} \longrightarrow X_{0} \times\left\{\left[f_{0}\right]\right\} \subset \mathcal{X}_{n}
$$

be the morphism that lifts the image $C_{0}$ to $\mathcal{X}_{n}$. The projection

$$
P: \mathcal{X}_{n} \longrightarrow S
$$

induces a map on the sections of bundles over $\mathbf{P}^{1}$,

$$
P^{s}: H^{0}\left(\bar{c}_{0}^{*}\left(T_{\mathcal{X}_{n}}\right)\right) \longrightarrow T_{\left[f_{0}\right]} S,
$$

where $T_{\left[f_{0}\right]} S \simeq H^{0}\left(T_{\left[f_{0}\right]} S \otimes \mathcal{O}_{\mathbf{P}^{1}}\right)$ is the space of global sections of the trivial bundle, each of whose fibre is $T_{\left[f_{0}\right]} S$. Observe the commutative diagram

$$
\begin{array}{ccc}
T_{\left(c_{0},\left[f_{0}\right]\right)} \Gamma & \stackrel{\left(e_{\Gamma}\right)_{*}}{\longrightarrow} & H^{0}\left(\bar{c}_{0}^{*}\left(T_{\mathcal{X}_{n}}\right)\right) \\
\downarrow\left(\pi_{\Gamma}\right)_{*} & & \downarrow P_{n}^{s} \\
T_{\left[f_{0}\right]} S & = & T_{\left[f_{0}\right]} S
\end{array}
$$

(see $(2.7)$ for $\left.P_{n}^{s}\right)$ where $\left(e_{\Gamma}\right)_{*}$ is induced from the differential of the evaluation $e_{\Gamma}$ :

$$
\begin{array}{ccc}
e_{\Gamma}: \Gamma \times \mathbf{P}^{1} & \longrightarrow & \mathcal{X}_{n} \\
(c,[f], t) & \longrightarrow & c(t) \times\{[f]\} .
\end{array}
$$

Since $f_{0}$ is generic and $\pi_{\Gamma}$ is dominant (by the assumption of the lemma), then $\left(c_{0},\left[f_{0}\right]\right) \in \Gamma$ is a generic point in $\Gamma_{0}$. Then the dominance of $\pi_{\Gamma}$ implies the surjectivity of $\left(\pi_{\Gamma}\right)_{*}$. Thus, $P_{n}^{s}$ is surjective. By Lemma 2.1, we have proved Lemma 2.2.

3. The quotient bundle $c_{0}^{*}\left(T_{\mathbf{P}^{n}}\right) / E^{\prime}$. In this section, we prove the quotient bundle $c_{0}^{*}\left(T_{\mathbf{P}^{n}}\right) / E^{\prime}$ is generated by a special type $V$ of global sections. Also, these special sections come from a particular type of first order deformation of hypersurfaces. Let us introduce a long setting that defines this special type of section. 
3.1. A parameter space of hypersurfaces. Let $S \simeq C^{N}$ be an affine open set of $\mathbf{P}\left(H^{0}\left(\mathcal{O}_{\mathbf{P}^{n}}(h)\right)\right)$. Let $f_{0} \in S$. Specifically, we can let

$$
S=\left\{f_{0}+\sum_{i=1}^{N} a_{i} f_{i}\right\},
$$

where $\left\{f_{i}\right\}, i=0, \ldots, N$, is a basis for the linear space $H^{0}\left(\mathcal{O}_{\mathbf{P}^{n}}(h)\right)$, and $\left(a_{1}, \ldots, a_{N}\right)$ are coordinates of $S=\mathbf{C}^{N}$.

Let

$$
\mathcal{X} \subset \mathbf{P}^{n} \times \mathbf{S}
$$

and

$$
\mathcal{X}=\{(x,[f]): f(x)=0\}
$$

Let

$$
F(a, x)=f_{0}(x)+\sum_{i=1}^{N} a_{i} f_{i}(x), \quad a \in S, x \in \mathbf{P}^{n},
$$

be the corresponding universal polynomial that defines $\mathcal{X}_{n}$.

Definition 3.1. There is an identification of the tangent space at the origin $\left[f_{0}\right] \in S$ such that, at any point $[f]$ in general,

$$
T_{\left[f_{0}\right]} S=S=T_{[f]} S .
$$

More specifically, if $[f] \in S$ is a polynomial $\neq f_{0}, \vec{f}$ is defined to be the corresponding vector field in $T_{\left[f_{0}\right]} S$ via formula (3.4). So $\vec{f}$ represents the direction of the line connecting $\left[f_{0}\right]$ and $[f]$.

3.2. A quotient vector bundle via decomposition. Now we introduce a rational curve. Let $\bar{c}_{0}$ be the smooth embedding map from

$$
\begin{array}{ccc}
\bar{c}_{0}: \mathbf{P}^{1} & \bar{c}_{0} & \mathbf{P}^{n} \times\left\{\left[f_{0}\right]\right\} \subset \mathbf{P}^{n} \times S \\
t & \longrightarrow & \left(c_{0}(t),\left[f_{0}\right]\right)
\end{array}
$$

induced from $c_{0}$ in formula (1.1), and $\bar{C}_{0}=C_{0} \times\left\{\left[f_{0}\right]\right\}$. From now on, we assume that assumption (1.3) holds. By Lemma 2.1, $P_{n}^{s}$ is 
surjective. Thus, we can choose sections

$$
\beta_{i}, \quad i=1, \ldots, N
$$

in $H^{0}\left(\bar{c}_{0}^{*}(T \mathcal{X})\right)$ such that $P_{n}^{s}\left(\beta_{i}\right)=\partial / \partial a_{i}$. The inverse $\beta_{i}$ is clearly not unique, but we choose one for each $i$. Let $G$ be the subbundle generated by these $N$ sections $\beta_{i} \in H^{0}\left(\bar{c}_{0}^{*}(T \mathcal{X})\right)$. $G$ is not unique. We may choose $G$ so it is invariant under the PGL $(n+1)$ action (see [9]), i.e., $G$ contains all the vectors tangent to the orbit of PGL $(n+1)$ action on $\mathbf{P}^{n} \times S$. Because of $\partial / \partial a_{i}$, these sections $\beta_{i}$ form a direct pointwise sum in the bundle $\bar{c}_{0}^{*}(T \mathcal{X})$. Hence, $G$ is a trivial bundle of rank $N$. This bundle $G$ gives us the decompositions

$$
\begin{aligned}
\bar{c}_{0}^{*}\left(T_{\mathbf{P}^{n} \times S}\right) & \simeq\left(\oplus_{N} \mathcal{O}\right) \oplus \bar{c}_{0}^{*}\left(T_{\left(\mathbf{P}^{n} \times S\right) / S}\right), \\
\bar{c}_{0}^{*}\left(T_{\mathcal{X}}\right) & \simeq\left(\oplus_{N} \mathcal{O}\right) \oplus \bar{c}_{0}^{*}\left(T_{\mathcal{X} / S}\right),
\end{aligned}
$$

where $\mathcal{O}$ is the trivial line bundle on $\mathbf{P}^{1}$, and $\oplus_{N} \mathcal{O} \simeq G$. Thus, we have the isomorphisms $I_{1}$ and $I_{2}$ :

$$
\begin{aligned}
& I_{1}: \frac{\bar{c}_{0}^{*}\left(T_{\mathcal{X}}\right)}{G} \simeq \bar{c}_{0}^{*}\left(T_{\mathcal{X} / S}\right), \\
& I_{2}: \frac{\bar{c}_{0}^{*}\left(T_{\mathbf{P}^{n} \times S}\right)}{G} \simeq \bar{c}_{0}^{*}\left(T_{\left(\mathbf{P}^{n} \times S\right) / S}\right) .
\end{aligned}
$$

Then, we have the isomorphism $I_{3}$ :

$$
\frac{\bar{c}_{0}^{*}\left(T_{\mathcal{X}}\right) / G}{I_{1}^{*}\left(T_{\bar{C}_{0}}\right)} \simeq N_{C_{0} / X_{0}} \simeq \mathcal{O}_{\mathbf{P}^{1}}\left(k_{1}\right) \oplus \cdots \oplus \mathcal{O}_{\mathbf{P}^{1}}\left(k_{n-2}\right) .
$$

Let $k_{n-2}$ be the smallest among all $k_{i}, i=1, \ldots, n-2$. Let $E \subset \bar{c}_{0}^{*}\left(T_{\mathcal{X}}\right)$ be the inverse image of

$$
\mathcal{O}_{\mathbf{P}^{1}}\left(k_{1}\right) \oplus \cdots \oplus \mathcal{O}_{\mathbf{P}^{1}}\left(k_{n-3}\right)
$$

under the bundle morphism

$$
\bar{c}_{0}^{*}\left(T_{\mathcal{X}}\right) \quad \longrightarrow \quad N_{C_{0} / X_{0}} \longrightarrow \mathcal{O}_{\mathbf{P}^{1}}\left(k_{1}\right) \oplus \cdots \oplus \mathcal{O}_{\mathbf{P}^{1}}\left(k_{n-2}\right) .
$$

Also, let $E^{\prime}$ be the inverse of

$$
\mathcal{O}_{\mathbf{P}^{1}}\left(k_{1}\right) \oplus \cdots \oplus \mathcal{O}_{\mathbf{P}^{1}}\left(k_{n-3}\right)
$$

under the bundle morphism

$$
\bar{c}_{0}^{*}\left(T_{X_{0}}\right) \quad \longrightarrow N_{C_{0} / X_{0}} \longrightarrow \mathcal{O}_{\mathbf{P}^{1}}\left(k_{1}\right) \oplus \cdots \oplus \mathcal{O}_{\mathbf{P}^{1}}\left(k_{n-2}\right)
$$


Then we have

$$
\frac{\bar{c}_{0}^{*}\left(T_{\mathbf{P}^{n} \times S}\right)}{E} \simeq \mathcal{O}_{\mathbf{P}^{1}}\left(d_{1}\right) \oplus \mathcal{O}_{\mathbf{P}^{1}}\left(d_{2}\right) \simeq c_{0}^{*}\left(T_{\mathbf{P}^{n}}\right) E^{\prime}
$$

where

$$
d_{1}+d_{2}=(n+1) d-2-\sum_{i=1}^{n-3} k_{i}, d_{1} \leq d_{2} .
$$

Let $D$ be the inverse image of the summand $\mathcal{O}_{\mathbf{P}^{1}}\left(d_{2}\right)$ under the map

$$
\bar{c}_{0}^{*}\left(T_{\mathbf{P}^{n} \times S}\right) \quad \longrightarrow \mathcal{O}_{\mathbf{P}^{1}}\left(d_{1}\right) \oplus \mathcal{O}_{\mathbf{P}^{1}}\left(d_{2}\right) .
$$

( $D$ is most likely unique depending on whether inequalities for $d_{2}, k_{n-2}$ are strict. But the uniqueness of $D$ will not affect the proof. So we fix D.)

3.3. A property on the global generation of the quotient bundle. Let $\theta=\left\{p_{1}, \ldots, p_{m}\right\}$ be an element in the symmetric product $S y^{m}\left(\mathbf{P}^{1}\right)$,

$$
0 \leq m<\mu\left(C_{0}\right)-1 \text {, }
$$

where $S y^{m}$ is the symmetric product. Let $H_{\theta}^{0}\left(\mathcal{O}_{\mathbf{P}^{n}}(1)\right)$ be the sublinear system of $H^{0}\left(\mathcal{O}_{\mathbf{P}^{n}}(1)\right)$ with base points $p_{i}, i=1, \ldots, m$, i.e.,

$$
H_{\theta}^{0}\left(\mathcal{O}_{\mathbf{P}^{n}}(1)\right)=H^{0}\left(\mathcal{O}_{\mathbf{P}^{n}}(1) \otimes \mathcal{I}_{p_{1}} \otimes \cdots \otimes \mathcal{I}_{p_{m}}\right) .
$$

Let

$$
V=\bigcup_{\theta \in S y^{m}\left(\mathbf{P}^{1}\right)} \operatorname{Sym}^{h}\left(H_{\theta}^{0}\left(\mathcal{O}_{\mathbf{P}^{n}}(1)\right)\right)
$$

where $\mathrm{Sym}^{h}$ is the $h$ th symmetric algebra of a vector space.

\section{Lemma 3.2.}

$$
\operatorname{span}(V)=H^{0}\left(\mathcal{O}_{\mathbf{P}^{n}}(h)\right) .
$$

Proof. Consider the Veronese map $v_{h}$

$$
\begin{array}{ccc}
H^{0}\left(\mathcal{O}_{\mathbf{P}^{n}}(1)\right) & \stackrel{v_{h}}{\longrightarrow} & H^{0}\left(\mathcal{O}_{\mathbf{P}^{n}}(h)\right) \\
f & \longrightarrow & f^{h} .
\end{array}
$$


Since the Veronese variety is non-degenerated, the linear span of its image is the entire space, i.e.,

$$
\operatorname{span}\left(v_{h}\left(H^{0}\left(\mathcal{O}_{\mathbf{P}^{n}}(1)\right)\right)\right)=H^{0}\left(\mathcal{O}_{\mathbf{P}^{n}}(h)\right) .
$$

By the definition,

$$
\operatorname{span}\left(v_{h}\left(H^{0}\left(\mathcal{O}_{\mathbf{P}^{n}}(1)\right)\right)\right) \subset \operatorname{span}(V) .
$$

Thus,

$$
\operatorname{span}(V)=H^{0}\left(\mathcal{O}_{\mathbf{P}^{n}}(h)\right)
$$

This completes the proof.

Proposition 3.3. If assumption (1.3) holds, V, identified with the subspace

$$
\{0\} \oplus V \subset \bar{c}_{0}^{*}\left(T_{\mathbf{P}^{n} \times S}\right),
$$

via formula (3.4), generates the bundle

$$
\frac{\bar{c}_{0}^{*}\left(T_{\mathbf{P}^{n} \times S}\right)}{E} \simeq \frac{c_{0}^{*}\left(T_{\mathbf{P}^{n}}\right)}{E^{\prime}}
$$

after modulo $E$.

To prove this lemma, we need to prove the following.

Lemma 3.4. If assumption (1.3) holds, the global sections of subbundle

$$
\bar{c}_{0}^{*}\left(T_{\left.\left(\mathbf{P}^{n} \times S\right) / \mathbf{P}^{n}\right) \quad(\text { modulo } E)}\right.
$$

generate the bundle $\bar{c}_{0}^{*}\left(T_{\mathbf{P}^{n} \times S}\right) / E \simeq c_{0}^{*}\left(T_{\mathbf{P}^{n}}\right) / E^{\prime}$.

Proof. There is a PGL $(n+1)$ action on $\mathbf{P}^{n}$. This action induces a PGL $(n+1)$ action on

$$
\mathbf{P}^{N}=\mathbf{P}\left(\mathcal{O}_{\mathbf{P}^{n}}(h)\right) .
$$

Thus, there is a PGL $(n+1)$ action on $\mathbf{P}^{n} \times \mathbf{P}^{N}$,

$$
g(f, x)=(g(f), g(x))=\left(f\left(g^{-1}(x)\right), g(x)\right) .
$$

If $z=\left(x,\left[f_{0}\right]\right) \in \bar{C}_{0}$, infinitesimally we have a linear map $\alpha_{z}$,

$$
\begin{array}{ccc}
T_{I d}(\operatorname{PGL}(n+1)) & \stackrel{\alpha_{z}}{\longrightarrow} & T_{z}\left(\mathbf{P}^{N} \times \mathbf{P}^{n}\right) \\
g & \longrightarrow & \left(g\left(f_{0}\right), g(x)\right) .
\end{array}
$$


Because $G$ is invariant under PGL $(n+1)$ action, then

$$
\text { image }\left.\left(\alpha_{z}\right) \in G\right|_{z} \text {. }
$$

Let

$$
W=\left\{g\left(f_{0}\right): g \in T_{\mathrm{Id}}(\operatorname{PGL}(n+1))\right\} \subset T_{f_{0}} \mathbf{P}^{N} .
$$

It is clear that $\alpha_{z}\left(T_{\mathrm{Id}}(\mathrm{PGL}(n+1))\right)$ is projected onto $T_{x} \mathbf{P}^{n}$. Hence,

$$
\{0\} \oplus W \subset H^{0}\left(\bar{c}_{0}^{*}\left(T_{\mathbf{P}^{n} \times S / \mathbf{P}^{n}}\right)\right) \quad \text { (modulo } E \text { ) }
$$

generates the bundle

$$
\frac{\bar{c}_{0}^{*}\left(T_{\mathbf{P}^{n} \times S}\right)}{E}=\bar{c}_{0}^{*}\left(\frac{T_{\mathbf{P}^{n} \times S / S}}{\bar{C}_{0}}\right) \simeq \frac{c_{0}^{*}\left(T_{\mathbf{P}^{n}}\right)}{E^{\prime}} .
$$

The proof is complete.

Proof of Proposition 3.3. By Lemma 3.2, $V$ linearly spans $H^{0}\left(\mathcal{O}_{\mathbf{P}^{n}}(h)\right)$. Using the identification in (3.4), $\{0\} \oplus V$ spans

$$
H^{0}\left(\bar{c}_{0}^{*}\left(T_{\left(\mathbf{P}^{n} \times S\right) / \mathbf{P}^{n}}\right)\right) .
$$

Then Lemma 3.4 says the global sections of

$$
\left.\left.\bar{c}_{0}^{*}\left(T_{\left(\mathbf{P}^{n} \times S\right) / \mathbf{P}^{n}}\right)\right) \quad \text { (modulo } E\right)
$$

generate $\bar{c}_{0}^{*}\left(T_{\mathbf{P}^{n} \times S}\right) / E$, then so does $V$. This completes the proof.

\section{The proof of Theorem 1.1 .}

\section{Proof.}

(i) We show the proof by a contradiction. So, suppose otherwise. Such a rational curve $c_{0}$ exists on the smooth $X_{0}$. Then

$$
m\left(\frac{2 h+1}{h+1}\right)<d<\frac{2+m(n-2)}{2 n-h-1}, \quad 0 \leq m<\mu\left(C_{0}\right)-1,
$$

where $d=\operatorname{deg}\left(C_{0}\right)$. By Lemma 2.1, the map $P^{s}$ is surjective. Then we can apply Proposition 3.3, which says that the set of sections in the form

$$
L_{1} \cdots L_{h}, \quad L_{i} \in H_{\theta}^{0}\left(\mathcal{O}_{\mathbf{P}^{n}}(1)\right)
$$

generates the bundle $\bar{c}_{0}^{*}\left(T_{\mathbf{P}^{n} \times S}\right) / E$. Since $D$ is a proper subbundle of $\bar{c}_{0}^{*}\left(T_{\mathbf{P}^{n} \times S}\right)$, Proposition 3.3 implies that there exist generic sections 
$L_{0}, \ldots, L_{h} \in H_{\theta}^{0}\left(\mathcal{O}_{\mathbf{P}^{n}}(1)\right)$, such that the $c_{0}^{*}\left(L_{i}\right), c_{0}^{*}\left(L_{j}\right)$ for $i \neq j$ do not have common zeros except that $p_{1}, \ldots, p_{m} \in \theta$ with multiplicity 1 ,

$$
\bar{c}_{0}^{*}\left(\overrightarrow{L_{0} \cdots L_{h-1}}\right)
$$

is a non-zero section that does not lie in $D$, where $\overrightarrow{L_{0} \cdots L_{h-1}}$ represents the direction of the line connecting $f_{0}$ and $L_{0} \cdots L_{h-1}$ in $S$ (see Definition 3.1). We should note that, in particular, the section $\bar{c}_{0}^{*}\left(\overrightarrow{L_{0} \cdots L_{h-1}}\right)$ does not lie in $E$.

Next, we show that the numerical condition (4.1) forces

$$
\bar{c}_{0}^{*}\left(\overrightarrow{L_{0} \cdots L_{h-1}}\right)
$$

to lie in $D$.

Using the chosen sections $L_{0}, \ldots, L_{h}$, we construct vector fields

$$
u_{i}=L_{h} \frac{\partial}{\partial a_{h}}-L_{i} \frac{\partial}{\partial a_{i}}, \quad i=0, \ldots, h-1,
$$

on $S \times \mathbf{C}^{n+1}$, where

$$
\frac{\partial}{\partial a_{i}}=\overrightarrow{L_{0} \cdots \widehat{L}_{i} \cdots L_{h}}
$$

as in Definition 3.1, are the tangent fields on $S$. The $u_{i}$ annihilate universal polynomial $F$ that defines the universal hypersurface $\mathcal{X}$. Hence, $u_{i}$ are sections of the bundle

$$
T_{\mathcal{X}} \otimes \mathcal{O}_{\mathbf{P}^{n}}(1) .
$$

Let $v_{i}=\bar{c}_{0}^{*}\left(u_{i}\right)$ be the pull-back of $u_{i}$ to $\mathbf{P}^{1}$. The non-zero nature of sections $v_{i}$ is the key to the proof of Theorem 1.1. Notice that $v_{i}$ must lie in $\bar{c}_{0}^{*}\left(T_{\mathcal{X}}(1)\right)$. Using formula $(3.8), v_{i}$ is reduced to sections of

$$
\left.\frac{\bar{c}_{0}^{*}\left(T_{\mathcal{X}}\right) / G}{I_{1}^{*}\left(T_{\bar{C}_{0}}\right)} \otimes \mathcal{O}_{\mathbf{P}^{1}}(d) \simeq\left((E / G) \otimes \mathcal{O}_{\mathbf{P}^{1}}(d)\right)\right) \oplus \mathcal{O}_{\mathbf{P}^{1}}\left(k_{n-2}+d\right) .
$$

Next, we consider the bound for $k_{n-2}+d$. By the result of $[\mathbf{1 1}]^{2}$, $2 n-h-1 \geq 1$. So, the condition $d<2+m(n-2) /(2 n-h-1)$ implies that

$$
(n+1) d-h d-2<-(n-2)(d-m) .
$$


The left hand side of formula (4.3) is

$$
c_{1}\left(N_{C_{0} / X_{0}}\right)=k_{1}+\cdots+k_{n-2} .
$$

Since $k_{n-2}$ is the smallest among $k_{1}, \ldots, k_{n-3}, k_{n-2}$, $(n-2) k_{n-2} \leq k_{1}+\cdots+k_{n-2}=(n+1) d-h d-2<-(n-2)(d-m)$. Hence, $k_{n-2}<-(d-m)$. Then,

$$
k_{n-2}+d<-(d-m)+d=m .
$$

Since $v_{i}$ has $m$ zeros at $p_{1}, \ldots, p_{m}$, by the bound of $k_{n-2}+d, v_{i}$ must be in $E$ along $\mathbf{P}^{1}$. Then formula (4.2) says that $\bar{c}_{0}^{*}\left(\partial / \partial a_{h}\right)$ must lie in $E$ at the zeros of $c_{0}^{*}\left(L_{i}\right), i=0, \ldots, h-1$, other than $p_{1}, \ldots, p_{m}$, because $c_{0}^{*}\left(L_{h}\right)$ does not vanish at any of the zeros of $c_{0}^{*}\left(L_{i}\right)$ along $\mathbf{P}^{1}$ except at $p_{1}, \ldots, p_{m}$. Thus, $\bar{c}_{0}^{*}\left(\partial / \partial a_{h}\right)$ does not lie in $E$ (by the choice of $\left.L_{i}\right)$, but lies in $E$ at least at $h(d-m)$ points. At last, we see this is impossible because

$$
\bar{c}_{0}^{*}\left(T_{\mathbf{P}^{n} \times S}\right) / E \simeq \mathcal{O}_{\mathbf{P}^{1}}\left(d_{1}\right) \oplus \mathcal{O}_{\mathbf{P}^{1}}\left(d_{2}\right),
$$

where $d_{1}+d_{2}=h d+k_{n-2}$ and $d_{1} \leq d_{2}$. Then, $d_{1}$ has the following bound (because of the inequality $m(2 h+1 / h+1)<d$ and formula $(4.4)$ ),

$$
h(d-m)>\frac{h d-(d-m)}{2} \geq \frac{h d+k_{n-2}}{2} \geq d_{1} .
$$

Because of this bound, $\bar{c}_{0}^{*}\left(\partial / \partial a_{h}\right)$ modulo $E$ must lie entirely in the summand $\mathcal{O}_{\mathbf{P}^{1}}\left(d_{2}\right)$. Recall that $D$ is the sub-bundle in $\bar{c}_{0}^{*}\left(T_{\mathbf{P}^{n} \times S}\right)$ such that $D / E=\mathcal{O}_{\mathbf{P}^{1}}\left(d_{2}\right)$. Thus, $\bar{c}_{0}^{*}\left(\partial / \partial a_{h}\right)$ must lie in $D$. This contradicts our choice of

$$
\frac{\partial}{\partial a_{h}}=\overrightarrow{L_{0} \cdots L_{h-1}},
$$

which says that $\bar{c}_{0}^{*}\left(\overrightarrow{L_{0} \cdots L_{h-1}}\right)$ does not lie in $D$. This completes the proof for the first part.

(ii) This part follows from the first part because of the exact sequence (1.2).

(iii) If $X_{0}$ is a generic hypersurface containing a smooth rational curve $C_{0}$, then using Lemma 2.2, we obtain that assumption (1.3) holds. Thus, all results in part (i) hold. 
5. Examples. We give three examples that follow from Theorem 1.1. The first two, to our knowledge, cannot be derived from previously known results, but the last one can.

Example 5.1. There are no quadratic curves in a general hypersurface of degree 14 in $\mathbf{P}^{9}$. This is a direct consequence of Theorem 1.1 for $n=9, h=14$ and $d=2$.

Example 5.2. There are no irreducible, rational quartic curves in a general hypersurface of degree 54 in $\mathbf{P}^{30}$. This is a direct consequence of Theorem 1.1 for $n=30, h=54$ and $d=4$.

Example 5.3. The condition $H^{1}\left(N_{C_{0} / X_{0}}\right)=0$ is actually much stronger than assumption (1.3). Therefore, the application of part (2) of Theorem 1.1 may be limited. Let us observe one in the following. Consider an irreducible quadratic curve $C_{0}$ in a smooth sextic threefold $X_{0}$ (which is non generic). Then its normal bundle $N_{C_{0} / X_{0}}$ cannot have the most balanced splitting

$$
\mathcal{O}_{\mathbf{P}^{1}}(-1) \oplus \mathcal{O}_{\mathbf{P}^{1}}(-1),
$$

because, otherwise, $H^{1}\left(N_{C_{0} / X_{0}}\right)=0$, and by Theorem 1.1, the degree of $C_{0}$ cannot be 2 .

This result can be obtained by using other methods. For instance, consider the combination of results of $[\mathbf{7}, \mathbf{9}, \mathbf{1 0}]$ in the following. If $H^{1}\left(N_{C_{0} / X_{0}}\right)=0$, Kodaira's theorem says that $C_{0}$ can globally deform to all sextics. Then, a generic sextic threefold contains a rational curve. On the other hand, since, in this case, $\operatorname{deg}\left(X_{0}\right) \geq 2 n-2$, Voisin's result says that a generic sextic does not contain a rational curve. This is a contradiction.

\section{ENDNOTES}

1. The references listed here are not complete. We apologize for this incompleteness due to the quantity of papers in this area.

2. We cannot use Voisin's result in [9] because $X_{0}$ in Theorem 1.1 is not generic. 


\section{REFERENCES}

1. H. Clemens, Curves in generic hypersurfaces, Ann. Sci. École Norm. 19 (1986), 629-636.

2. _ Some results on Abel-Jacobi mappings, in Topics in transcendental algebraic geometry, Princeton University Press, Princeton, 1984.

3. , Homological equivalence, modulo algebraic equivalence, is not finitely generated, Publ. Math IHES 58 (1983), 19-38.

4. L. Chiantini, A.F. Lopez and Z. Ran, Subvarieties of generic hypersurfaces in any variety, Math. Proc. Camb. Phil. Soc. 130 (2001), 259-268.

5. L. Ein, Subvarieties of generic complete intersections, Invent. Math. 94 (1988), 163-169.

6. S. Katz, On the finiteness of rational curves on quintic threefolds, Comp. Math. 60 (1986), 151-162.

7. K. Kodaira, On stability of compact submanifolds of complex manifolds, Amer. J. Math. 85 (1963), 79-94.

8. G. Pacienza, Rational curves on general projective hypersurfaces, J. Alg. Geom. 12 (2003), 471-476.

9. C. Voisin, On a conjecture of Clemens on rational curves on hypersurfaces, J. Differ. Geom. 44 (1996), 200-213.

10. , A correction: "On a conjecture of Clemens on rational curves on hypersurfaces," J. Differ. Geom. 49 (1998), 601-611.

11. B. Wang, Obstructions to the deformations of curves to other hypersurfaces, arXiv:1110.0184v3, 2011.

12. G. Xu, Subvarieties of general hypersurfaces in projective space, J. Differ. Geom. 39 (1994), 139-172.

Mathematics and Computer Science Department, Rhode Island College, 600 Mount pleasant Ave., Providence, RI 02908

Email address: binwang64319@gmail.com 